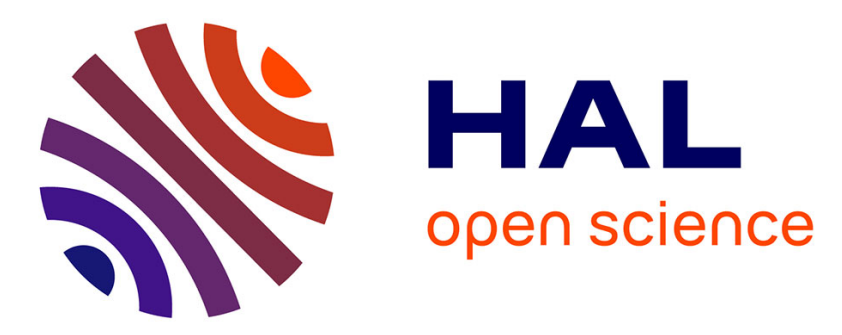

\title{
Calibration of a magnetometer array using motion capture equipment
}

Charles-Ivan Chesneau, Rémi Robin, Hendrik Meier, Mathieu Hillion, Christophe Prieur

\section{- To cite this version:}

Charles-Ivan Chesneau, Rémi Robin, Hendrik Meier, Mathieu Hillion, Christophe Prieur. Calibration of a magnetometer array using motion capture equipment. Asian Journal of Control, 2019, 21 (4), pp.1459-1469. 10.1002/asjc.2043 . hal-02368023

\section{HAL Id: hal-02368023 https://hal.science/hal-02368023}

Submitted on 18 Mar 2020

HAL is a multi-disciplinary open access archive for the deposit and dissemination of scientific research documents, whether they are published or not. The documents may come from teaching and research institutions in France or abroad, or from public or private research centers.
L'archive ouverte pluridisciplinaire HAL, est destinée au dépôt et à la diffusion de documents scientifiques de niveau recherche, publiés ou non, émanant des établissements d'enseignement et de recherche français ou étrangers, des laboratoires publics ou privés. 


\title{
Calibration of a magnetometer array using motion capture equipment
}

\author{
Charles-Ivan CHESNEAU, Rémi ROBIN, Hendrik MEIER, Mathieu HILLION, Christophe PRIEUR
}

\begin{abstract}
This paper studies the problem of calibrating an array of singleaxis magnetometers in an unknown static inhomogeneous magnetic field using motion capture equipment. A proof of identifiability is given, practical identifiability of calibration parameters is established in simulation, and real world experiments are conducted to demonstrate the feasibility of this approach. Unlike many techniques of the state of the art, the proposed solution does not require a homogeneous field, as in fact, we demonstrate that an inhomogeneous field enlarges the set of identifiable parameters. Under the above-mentioned assumptions, this approach may be used to extend selfcalibration techniques of visual-inertial setups to magnetic sensor arrays in indoor environments.
\end{abstract}

\section{Introduction}

Precise calibration of micro-electronic singleaxis point-magnetometers is often required for practical applications, which has motivated research for suitable calibration techniques. Several magnetometer calibration techniques have been developed using the earth magnetic field $[1,2,3,4]$, the most frequently mentioned being ellipsoid fitting. These techniques require a homogeneous and static magnetic field to achieve a good calibration precision, which implies manual outdoor data collection or expensive setups. Other techniques exploit gyrometers [5], or coilgenerated homogeneous magnetic fields $[6,7,8]$, or coil-generated inhomogeneous magnetic fields [9, 10, 11]. Strictly speaking, because calibration processes involving homogeneous magnetic fields do not give access to sensor effective positions, magnetic field gradiometers that rely on the knowledge of such

Charles-Ivan Chesneau is with Sysnav, 57 rue de Montigny, 27200 Vernon, France, and Univ. Grenoble Alpes, CNRS, Grenoble INP, GIPSA-lab, F-38000 Grenoble, France charles-ivan. chesneaudsysnav. fr.

Rémi Robin, Hendrik Meier and Mathieu Hillion are with Sysnav.

Christophe Prieur is with Univ. Grenoble Alpes, CNRS, Grenoble INP, GIPSA-lab, christophe.prieur@gipsa-lab. fr

The authors would like to thank David Caruso, for fruitful discussions that helped setting up this work. positions cannot be calibrated this way. Among the techniques involving inhomogeneous magnetic fields, the one discussed in [9] requires accurate manipulations, and $[10,11]$ describe a simple process for calibrating SQUID (Superconducting Quantum Interference Device) magnetometers, but require that sensors be sensitive enough to measure the generated magnetic field. The problem of precisely calibrating an array of single-axis magnetometers is still not satisfactorily solved in order to be applicable in socalled magneto-inertial dead-reckoning applications, which in general do not use SQUID magnetometers. This observation motivates the research of a calibration technique that is less demanding on the environment and on dedicated setups.

In the last decade, several papers have shown the feasibility of the above-mentioned navigation technique of magneto-inertial dead-reckoning. Its theoretical viability was shown in [12], the technique was patented in [13], further studied in theory and practice in the context of pedestrian indoor navigation in [14, 15, 16]. Its potential for space applications were studied in [17]. Recently, Refs. [18, 19] studied indoor navigation performance achievable with this technique alone in various environment including an industrial facility, by using Extended Kalman Filters taking into account various sources of error. At the same time, Refs. [20, 21, 22] provided several frameworks in which magnetic sensor information can be combined with 
visual odometry, resulting in a robust dead-reckoning navigation solution.

In short, magneto-inertial dead-reckoning consists of two steps: integration of strapdown IMU (Inertial Measurement Unit) navigation equations and correction of the estimated motion using a stationary inhomogeneous magnetic field and its spatial variations. Indoor magnetic distortions have proved to be sufficient for dead-reckoning, provided that accurate magnetic measurements are available. However, the required accuracy is rather demanding with regard to magnetometer calibration.

This paper presents a calibration technique for magnetometer arrays solving a problem inverse to magneto-inertial dead-reckoning. Whereas the latter, applicable for an indoor stationary (inhomogeneous) magnetic field [19], allows us to obtain positions and attitudes as a function of the calibration parameters (and the raw magnetic data), the present method builds on raw magnetic data paired with motion capture data, i.e. position and attitude of the array of the moving sensor device, in order to estimate calibration parameters as well as the magnetic field. The only parameter that is not accessible through our method is the global scale factor that defines the unit of the magnetic field (and may be adjusted to Tesla or Gauss in a separate experiment if desired); in any case, the choice of such a unit does not intervene in magneto-inertial deadreckoning.

The paper is organized as follows. In Sec. II, we state the explicit problem addressed by this article. In Sec. III, we mathematically prove the identifiability of calibration parameters. Section IV shows, using simulated data, that calibration parameters are in practice identifiable. Finally, Section V shows the results of applying this technique in real-world experiments, and Sec. VI contains some concluding remarks and points out a natural perspective. The appendices collect some proofs of intermediate results.

\section{Problem statement}

The problem under consideration is the calibration of an array of linear-response single-axis point magnetometers strapped on a rigid body using motion information.

As in [19], let $\mathfrak{R}_{n}$ be a navigation frame, and $\mathfrak{R}_{b}$ a frame of reference moving with the rigid body. Coordinates of vectors in $\Re_{n}$ (resp. $\mathfrak{R}_{b}$ ) are denoted with the prescript ${ }^{\mathrm{n}}$ (resp. ${ }^{\mathrm{b}}$ ).

Let $\mathrm{M}$ be a fixed point of the rigid body defining the origin of $\mathfrak{R}_{b}$; by definition, its coordinates in body frame are ${ }^{\mathrm{b}} \mathbf{M} \doteq 0$. Assuming $\mathfrak{R}_{n}$ and $\mathfrak{R}_{b}$ are each equipped with an orthonormal basis, an attitude matrix $R$, belonging to the special orthogonal group $\mathrm{SO}(3)$, can be defined such that for any vector $\mathbf{v},{ }^{\mathrm{n}} \mathbf{v}=R .{ }^{\mathrm{b}} \mathbf{v}$. The rigid body motion is defined by the time-evolution of ${ }^{\mathrm{n}} \mathbf{M}(t)$ and $R(t)$ during an experiment.

Let $\mathbf{B}$ denote the magnetic field, which is assumed static in $\mathfrak{R}_{n}$. This means that ${ }^{\mathrm{n}} \mathbf{B}$ varies only as a function of the position ${ }^{\mathrm{n}} \mathbf{M}$,

$$
\begin{aligned}
{ }^{\mathrm{n}} \mathbf{B}: \mathbb{R}^{3} & \rightarrow \mathbb{R}^{3} \\
{ }^{\mathrm{n}} \mathbf{M} & \mapsto{ }^{\mathrm{n}} \mathbf{B}\left({ }^{\mathrm{n}} \mathbf{M}\right) .
\end{aligned}
$$

Let ${ }_{j}^{\mathrm{b}} \mathbf{p}$ denote the position of the $j$-th single-axis magnetic sensor in the moving reference frame $\mathfrak{R}_{b}$. The magnetic field at this point, and in $\Re_{b}$, is related to the magnetic field in the navigation frame $\mathfrak{R}_{n}$ as*.

$$
{ }^{\mathrm{b}} \mathbf{B}\left({ }_{j}^{\mathrm{b}} \mathbf{p}, t\right)=R(t)^{\top} \cdot{ }^{\mathrm{n}} \mathbf{B}\left({ }^{\mathrm{n}} \mathbf{M}(t)+R(t) \cdot{ }_{j}^{\mathrm{b}} \mathbf{p}\right) .
$$

As ${ }^{\mathrm{n}} \mathbf{M}$ and $R$ vary as a function of time, so does in general ${ }^{\mathrm{b}} \mathrm{B}$.

To complete our measurement model, we specify the direction and scale factor of the $j$-th single-axis magnetic sensor by a co-vector ("row vector") ${ }_{j}^{\mathrm{b}} \mathrm{a}$ and add a (scalar) bias ${ }_{j} b$. The full model for a measurement with result ${ }_{j} y$ then reads

$$
{ }_{j} y={ }_{j}^{\mathrm{b}} \mathbf{a} \cdot R^{\top} \cdot{ }^{\mathrm{n}} \mathbf{B}\left({ }^{\mathrm{n}} \mathbf{M}+R \cdot{ }_{j}^{\mathrm{b}} \mathbf{p}\right)+{ }_{j} b
$$

for each magnetic single-axis sensor $j$. It is implied that the co-vector ${ }_{j}^{\mathrm{b}} \mathrm{a}$, the bias ${ }_{j} b$, and the position vector ${ }_{j}^{\mathrm{b}} \mathbf{p}$ (which is a usual "column vector") are timeindependent.

The magnetic field ${ }^{\mathrm{n}} \mathbf{B}$ lives in an infinitedimensional vector space - the space of solutions to Maxwell's equations. In practice, it is typically possible to describe this field with sufficient accuracy in a finitedimensional subspace, using only a finite number of coordinates that we denote $\alpha_{i}$.

During a calibration, ${ }^{\mathrm{n}} \mathbf{M}(t)$ and $R(t)$ are varied as a function of time $t$ with $t_{i} \leq t \leq t_{f}$. The timedependency of these (6D) coordinates inflicts a timedependency on the measurement functions ${ }_{j} y$. In the situation of an experiment that allows us to measure all these quantities, ${ }^{\mathrm{n}} \mathbf{M}(t), R(t)$, and ${ }_{j} y(t)$ at each time $t$, $t_{i} \leq t \leq t_{f}$, we consider the following problems:

P1 Are parameters ${ }_{j}^{\mathrm{b}} \mathrm{a},{ }_{j} b$, and magnetic field coordinates $\alpha_{i}$ identifiable if the effective position parameters ${ }_{j}^{\mathrm{b}} \mathbf{p}$ are given?

\footnotetext{
${ }^{*}$ In fact, Eq. (1) is merely a (Galilean) transformation between the two reference frames [23, 6.10.A, 11.10]
} 
P2 Are parameters ${ }_{j}^{\mathrm{b}} \mathbf{a},{ }_{j} b,{ }_{j}^{\mathrm{b}} \mathbf{p}$, and magnetic field coordinates $\alpha_{i}$ identifiable?

Problem P1 implies that we have to know the effective position of each individual sensor in both $\mathfrak{R}_{b}$ and $\mathfrak{R}_{n}$. Problem P2 does not require us to possess this information, a situation that is closer to what is obtained in a motion capture experiment: such a setup locates visual markers, while sensors are typically hidden inside of a package, and their position is not precisely known with respect to markers. Furthermore, effective positions are needed in order to calibrate a magnetic gradiometer built from an array of sensors. Proving that parameters are identifiable in Problems $\mathrm{P} 1$ and P2 would be equivalent to proving that any parameterization to (2) is unique - a notion we clarify in the next section. At the same time, the mere existence of such a parameterization is guaranteed in experiment by the physics behind the measurement model.

\section{Identifiability}

In this section, we prove the identifiability of calibration parameters under "mathematically controlled" conditions that, as we argue, represent nevertheless the situation in realistic experiments.

\subsection{Notion of identifiability and assumptions}

We adopt the setting of Sec. II and consider a set of $m$ single-axis magnetometers that measure the projection of the magnetic field $\mathbf{B}$. The field $\mathbf{B}$ is assumed time-independent in our navigation frame $\mathfrak{R}_{n}$. The measurement of each magnetometer is physically modeled by a function ${ }_{j} y, j \in\{1,2, \ldots, m\}$, that assigns to a $6 \mathrm{D}$ position $\left({ }^{\mathrm{n}} \mathbf{M}, R\right)$ of the rigid body the magnetic field component along the axis of the $j$-th magnetometer,

$$
\begin{aligned}
{ }_{j} y: \mathbb{R}^{3} \times \mathrm{SO}(3) & \rightarrow \mathbb{R} \\
\left({ }^{\mathrm{n}} \mathbf{M}, R\right) & \mapsto{ }_{j}^{\mathrm{b}} \mathbf{a} \cdot R^{\top} \cdot{ }^{\mathrm{n}} \mathbf{B}\left({ }^{\mathrm{n}} \mathbf{M}+R \cdot{ }_{j}^{\mathrm{b}} \mathbf{p}\right)+{ }_{j} b .
\end{aligned}
$$

We note that for a reasonable sensor, ${ }_{j}^{\mathrm{b}} \mathbf{a} \neq 0$, which is what we thus assume in the following. The magnetic field ${ }^{\mathrm{n}} \mathbf{B}$ is assumed to satisfy Maxwell's equations for a source-free region of space (which in particular implies analyticity). Furthermore, ${ }^{\mathrm{n}} \mathbf{B}$ is assumed not to vanish identically and to feature at least one position in which all eigenvalues of its derivative (Jacobian matrix) are different from each other. This assumption is verified by any realistic magnetic field. ${ }^{\dagger}$

In experiment, all the parameters and the magnetic field are a priori unknown and need to be determined in calibration. Let us unite these parameters into one tuple $\theta$,

$$
\theta \doteq\left(\left({ }_{j} b\right),\left({ }_{j}^{\mathrm{b}} \mathbf{p}\right),\left({ }_{j}^{\mathrm{b}} \mathbf{a}\right),{ }^{\mathrm{n}} \mathbf{B}\right)
$$

where the notation $\left({ }_{j} b\right)=\left({ }_{j} b\right)_{j=1, \ldots, m}$ represents the tuple of biases for all $m$ magnetometers (and the same applies to $\left({ }_{j}^{\mathrm{b}} \mathbf{p}\right)$ and $\left.\left({ }_{j}^{\mathrm{b}} \mathbf{a}\right)\right)$. The space of all such $\theta$ will be denoted $\Theta$. Each element $\theta \in \Theta$ defines a set of $m$ measurement functions ${ }_{j} y$, Eq. (3).

We interpret identifiability as the ability to distinguish different tuples of parameters by their corresponding measurement functions. This means that if two tuples of parameters $\theta$ and $\theta^{\prime}$ in the parameter space $\Theta$ are different, $\theta \neq \theta^{\prime}$, then their corresponding measurement functions $\left({ }_{j} y\right)$ and $\left({ }_{j} y^{\prime}\right)$ should be different too. In other words, there should be at least one $6 \mathrm{D}$ position ${ }^{\mathrm{n}} \mathbf{M}, R$ of the rigid body for which at least one of the $m$ magnetometers, say magnetometer $j$, produces a different measurement result, i.e. ${ }_{j} y\left({ }^{\mathrm{n}} \mathbf{M}, R\right) \neq{ }_{j} y^{\prime}\left({ }^{\mathrm{n}} \mathbf{M}, R\right)$.

However, this notion of identifiability is a little too strong to hold, as revealed by a quick inspection of Eq. (3). In fact, the functions $\left({ }_{j} y\right)$ are invariant under simultaneous rescaling ${ }_{j}^{\mathrm{b}} \mathbf{a} \mapsto \lambda_{j}^{\mathrm{b}} \mathbf{a}$ and ${ }^{\mathrm{n}} \mathbf{B} \mapsto{ }^{\mathrm{n}} \mathbf{B} / \lambda$ for $\lambda \in \mathbb{R} \backslash\{0\}$, which corresponds just to the global scale factor that, as we mentioned in Sec. I, we cannot calibrate.

This invariance motivates the definition of an equivalence relation " $~$ " on $\Theta$, which identifies two elements $\theta, \theta^{\prime} \in \Theta$ if and only if there exists a $\lambda \in$ $\mathbb{R} \backslash\{0\}$ such that

$$
\begin{gathered}
{ }^{\mathrm{n}} \mathbf{B}^{\prime} \equiv{ }^{\mathrm{n}} \mathbf{B} / \lambda, \\
\forall j,{ }_{j} b^{\prime}={ }_{j} b,{ }_{j}^{\mathrm{b}} \mathbf{p}^{\prime}={ }_{j}^{\mathrm{b}} \mathbf{p},{ }_{j}^{\mathrm{b}} \mathbf{a}^{\prime}=\lambda{ }_{j}^{\mathrm{b}} \mathbf{a} .
\end{gathered}
$$

In this case, we write $\theta \sim \theta^{\prime}$. In terms of the equivalence relation " $\sim$ ", we aim to prove identifiability in the sense that for two tuples $\theta, \theta^{\prime} \in \Theta$, that are not equivalent, $\theta \nsim$ $\theta^{\prime}$, the measurement functions are able to distinguish

\footnotetext{
$\dagger$ In Eqs. (1) and (3), we consider for the sake of simple notations $\mathbb{R}^{3}$ as domain of our fields. In fact, we could also restrict ourselves to a bounded open subset without any loss of validity of our statement. In an actual experiment, the position ${ }^{\mathrm{n}} \mathbf{M}$ is restricted to vary within some (bounded) open subset $V \subset \mathbb{R}^{3}$. Also, it is clearly sufficient to consider position vectors ${ }_{j}^{\mathrm{b}} \mathbf{p}$ within a ball whose radius $r$ is of the order of the linear size of the device that contains all magnetometers. Then, the domain of the magnetic field ${ }^{\mathrm{n}} \mathbf{B}$ can also be restricted to an open bounded subset $\subset \mathbb{R}^{3}$, for instance the interior of the union of the balls of radius $r$ around all ${ }^{\mathrm{n}} \mathbf{M} \in V$. Physical source currents of the magnetic field are assumed to be outside this subset.
} 
them from each other at least at one $6 \mathrm{D}$ position for at least one magnetometer, i.e. if $\theta \nsim \theta^{\prime}$, there exists a tuple $\left(j,{ }^{\mathrm{n}} \mathbf{M}, R\right)$ such that ${ }_{j} y\left({ }^{\mathrm{n}} \mathbf{M}, R\right) \neq{ }_{j} y^{\prime}\left({ }^{\mathrm{n}} \mathbf{M}, R\right)$.

In a more formal reformulation, the notion of identifiability we wish to prove is injectivity of the mapping $\theta \mapsto\left({ }_{j} y\right)$ on the identification space $\Theta / \sim$, see, e.g., [24], Def. (9.11) p. 62. The mapping is defined by inserting a representative $\theta$ into Eq. (3). Here and in the following, we use the notation $\theta$ for a particular representative of equivalent parameter tuples also for its equivalence class in $\Theta / \sim$.

\subsection{Theorems of identifiability}

Adopting the assumptions and notations of the previous sections, we formulate the following theorem of identifiability that addresses the problem $\mathrm{P} 2$ raised in Sec. II:

Theorem III.1 For each tuple of parameters $\theta \in$ $\Theta / \sim$, there exists an open neighborhood $T \subset \Theta / \sim$, $\theta \in T$, on which the mapping $\theta \mapsto\left({ }_{j} y\right)$, defined by inserting $\theta$ into Eq. (3), is injective.

The restriction to an open neighborhood applies only to the effective sensor positions $\left({ }_{j}^{\mathrm{b}} \mathbf{p}\right)$. For biases $\left({ }_{j} b\right)$, injectivity holds globally. For the scale factors ${ }_{j}^{\mathrm{b}} \mathbf{a}$ and the magnetic field ${ }^{\mathrm{n}} \mathbf{B}$, no restriction of locality is imposed beyond the required properties discussed in the preceding section.

In special situations, for instance, if the magnetic field is fully described by an affine mapping, our proof allows immediately to lift the restriction to an open neighborhood of $\left({ }_{j}^{\mathrm{b}} \mathbf{p}\right)$ and the theorem holds globally.

As a corollary to Theorem III.1, we find

Corollary III.2 The mapping $\theta \mapsto\left({ }_{j} y\right)$, defined by inserting $\theta$ into Eq. (3), is injective on any subset of $\Theta / \sim$ in which the sensor positions $\left({ }_{j}^{\mathrm{b}} \mathbf{p}\right)$ are constant.

We note that this corollary, which addresses Problem P1, is in fact valid for arbitrary magnetic fields as long as they do not vanish identically. No requirement on its gradient is necessary. (In particular, the gradient would be allowed to be identically zero.)

\subsection{Proof of Theorem III.1}

We proceed by proving parameter by parameter that changes in any parameter of a given $\theta \in \Theta / \sim$ result in a different measurement ${ }_{j} y\left({ }^{\mathrm{n}} \mathbf{M}, R\right)$ for at least one $6 \mathrm{D}$ position $\left({ }^{\mathrm{n}} \mathbf{M}, R\right)$, regardless of possible simultaneous changes of other parameters whose identifiability has not yet been proven.

The proof is built on the (theoretical) availability of a trajectory that traverses the entire $6 \mathrm{D}$ position manifold while we can expect a suitably designed experimental trajectory to cover a large-enough and sufficiently diverse subset that is representative for the entire manifold.

\subsubsection{Biases}

The identifiability of the biases ${ }_{j} b$ follows from the following lemma.

Lemma III.3 Let $j \in\{1,2, \ldots, m\}$. Using the notation of the previous sections, for all ${ }^{\mathrm{n}} \mathrm{M}$, the integral of ${ }_{j} y\left({ }^{\mathrm{n}} \mathrm{M}, R\right)$ over $R \in \mathrm{SO}(3)$ yields

$$
\oint_{R \in \mathrm{SO}(3)}{ }_{j} y\left({ }^{\mathrm{n}} \mathbf{M}, R\right) \mathrm{d} R={ }_{j} b,
$$

where $\mathrm{d} R$ denotes the Haar measure of $\mathrm{SO}(3)$ that normalizes its volume to unity.

Proof See Appendix A.

Lemma III.3 implies that if two tuples of parameters $\theta$ and $\theta^{\prime}$ that contain different biases ${ }_{j} b$ and ${ }_{j} b^{\prime}$ for the $j$-th magnetometer, then the integral of Lemma III.3 on their respective measurement functions will yield different results, regardless of other parameters. Inevitably, the measurement functions must thus differ for at least one position. Therefore, biases are identifiable.

\subsubsection{Effective sensor positions}

Let $j \in\{1,2, \ldots, m\}$. Since the bias ${ }_{j} b$ has been proven identifiable (see the preceding Sec. 3.3.1), we can assume it to be zero, ${ }_{j} b=0$, without loss of generality.

As noted in the statement of Theorem III.1, we refrain from trying to prove global injectivity with respect to the sensor positions $\left({ }_{j}^{\mathrm{b}} \mathbf{p}\right)$ and content ourselves with local injectivity around its true value.

Let $\theta_{j}=\left({ }_{j}^{\mathrm{b}} \mathbf{p},{ }_{j}^{\mathrm{b}} \mathbf{a},{ }^{\mathrm{n}} \mathbf{B}\right)$ denote the tuple of the true parameters of the physical model. The reader may assume that we have already "guessed" the true ${ }_{j}^{b} \mathbf{p}$, yet we are still unaware of its (local) uniqueness.

As to the scale factor ${ }_{j}^{\mathrm{b}} \mathbf{a}$ and the magnetic field ${ }^{\mathrm{n}} \mathbf{B}$, the only information we need (and have) is that by assumption, ${ }_{j}^{\mathrm{b}} \mathbf{a} \neq 0$ and that there is at least one 
position ${ }^{\mathrm{n}} \mathbf{M}_{0}$ in which the gradient of the magnetic field (Jacobian matrix),

$$
\left.\nabla^{\mathrm{n}} \mathbf{B}\right|_{{ }^{{ }} \mathbf{M}_{0}}=\left.\frac{\partial\left({ }^{\mathrm{n}} \mathbf{B}\right)}{\partial\left({ }^{\mathrm{n}} \mathbf{M}\right)}\right|_{{ }^{\mathrm{M}} \mathbf{M}={ }^{\mathrm{n}} \mathbf{M}_{0}},
$$

possesses eigenvalues that are all mutually different.

If, using only the knowledge of the measurement function ${ }_{j} y$, we can construct an injective mapping $\zeta$ from the effective positions space $\mathbb{R}^{3}$ into some other vector space such that $\zeta\left({ }_{j}^{\mathrm{b}} \mathbf{p}\right)=0$ (independently from ${ }_{j}^{\mathrm{b}} \mathbf{a}$ and ${ }^{\mathrm{n}} \mathbf{B}$ as long as the assumptions stated above hold), uniqueness and thus identifiability of ${ }_{j}^{b} \mathbf{p}$ is proven.

Lemma III.4 Under the assumptions made for ${ }_{j}^{\mathrm{b}} \mathrm{a}$ and ${ }^{\mathrm{n}} \mathbf{B}$, the construction of a mapping $\zeta$ as described above is possible in an open neighborhood of ${ }_{j}^{\mathrm{b}} \mathbf{p}$.

Proof In Appendix B, we present an explicit construction of a mapping $\zeta: \mathbb{R}^{3} \rightarrow \mathbb{R}^{6}$ using only knowledge of ${ }_{j} y$.

Remarks In the special case that the Jacobian matrix of the magnetic field is constant, i.e. if the magnetic field is affine, the arguments provided in Appendix B are sufficient to prove global identifiability.

\subsubsection{Scale factors and magnetic field}

Without loss of generality, $\theta$ is such that biases ${ }_{j} b$ are zero and that effective sensor positions $\left({ }_{j}^{\mathrm{b}} \mathbf{p}\right)$ are known. Also, by assumption, there exists a position ${ }^{\mathrm{n}} \mathbf{M}_{0}$ at which the magnetic field is non-zero, ${ }^{\mathrm{n}} \mathbf{B}\left({ }^{\mathrm{n}} \mathbf{M}_{0}\right) \neq 0$. In this situation, we can place any sensor, say the $j$-th, at this position ${ }^{\mathrm{n}} \mathbf{M}_{0}$ (in navigation frame) for an arbitrary orientation $R$. This is possible by choosing

$$
{ }^{\mathrm{n}} \mathbf{M}={ }^{\mathrm{n}} \mathbf{M}_{0}-R \cdot{ }_{j}^{\mathrm{b}} \mathbf{p} .
$$

As a result, a (theoretical) calibration experiment allows us to gain full knowledge of the auxiliary measurement function

$$
\begin{aligned}
{ }_{j} Y\left({ }^{\mathrm{n}} \mathbf{M}_{0}\right) & : R \mapsto{ }_{j} y\left({ }^{\mathrm{n}} \mathbf{M}_{0}-R \cdot{ }_{j}^{\mathrm{b}} \mathbf{p}, R\right) \\
& =\operatorname{Tr}\left[R^{\top} \cdot{ }^{\mathrm{n}} \mathbf{B}\left({ }^{\mathrm{n}} \mathbf{M}_{0}\right) \otimes{ }_{j}^{\mathrm{b}} \mathbf{a}\right],
\end{aligned}
$$

where ${ }_{j}^{\mathrm{b}} \mathbf{a}$ and ${ }^{\mathrm{n}} \mathbf{B}\left({ }^{\mathrm{n}} \mathbf{M}_{0}\right)$ denote the true (yet until now unknown to be unique) values for the scale vector and the magnetic field at ${ }^{\mathrm{n}} \mathbf{M}_{0}$; the symbol $\mathrm{Tr}$ is the trace.

We observe that

$$
A \mapsto \operatorname{Tr}\left[A^{\top} \cdot{ }^{\mathrm{n}} \mathbf{B}\left({ }^{\mathrm{n}} \mathbf{M}_{0}\right) \otimes{ }_{j}^{\mathrm{b}} \mathbf{a}\right]
$$

defines a linear form on the space of real $3 \times$ 3 matrices $\mathbb{R}^{3 \times 3}$ whose uniquely defined (standard) gradient is the matrix ${ }^{\mathrm{n}} \mathbf{B}\left({ }^{\mathrm{n}} \mathbf{M}_{0}\right) \otimes{ }_{j}^{\mathrm{b}} \mathbf{a}$. Since $\mathrm{SO}(3)$ as generating set in $\mathbb{R}^{3 \times 3}$ spans the entire matrix space, the knowledge of ${ }_{j} Y\left({ }^{\mathrm{n}} \mathbf{M}_{0}\right)$ for matrices $R \in \mathrm{SO}(3)$ is sufficient to uniquely determine ${ }^{\mathrm{n}} \mathbf{B}\left({ }^{\mathrm{n}} \mathbf{M}_{0}\right) \otimes{ }_{j}^{\mathrm{b}} \mathbf{a}$.

The rank of ${ }^{\mathrm{n}} \mathbf{B}\left({ }^{\mathrm{n}} \mathbf{M}_{0}\right) \otimes{ }_{j}^{\mathrm{b}} \mathbf{a}$ is 1 , lines are proportional to (the row vector) ${ }_{j}^{\mathrm{b}} \mathbf{a}$, columns are proportional to (the column vector) ${ }^{\mathrm{n}} \mathbf{B}\left({ }^{\mathrm{n}} \mathbf{M}_{0}\right)$, which means that ${ }_{j}^{\mathrm{b}} \mathbf{a}$ is identifiable up to an unknown scale factor $\lambda$ while the magnetic field at ${ }^{\mathrm{n}} \mathbf{M}_{0}$ is identifiable up to an unknown scale factor $1 / \lambda$.

Once ${ }^{n} \mathbf{B}\left({ }^{\mathrm{n}} \mathbf{M}_{0}\right)$ is identified up to the unknown scale factor $1 / \lambda$, all scale factors of the other sensors ${ }_{j^{\prime}}^{\mathrm{b}} \mathbf{a}$ for $j^{\prime} \neq j$ are identifiable (relatively to a chosen scale factor for ${ }_{j^{\prime}}^{\mathrm{b}} \mathbf{a}$ ), using the same construction.

Since this construction works at all positions ${ }^{\mathrm{n}} \mathbf{M}_{0}$ with non-zero magnetic field, the magnetic field itself is identifiable as well (up to an unknown scale factor $1 / \lambda$ ). This concludes the proof of Theorem III.1.

\subsection{Conclusion of the proof and discussion}

The above reasoning proves that Theorem III.1 and its Corollary III. 2 hold. Theorem III.1 states that unknown parameters corresponding to Problem P2 (defined in Sec. II) are locally identifiable, as long as there exists at least one place where the magnetic gradient is non-singular, and if all magnetometer scale factors ${ }_{j}^{\mathrm{b}} \mathrm{a}$ are different from zero. According to the remark at the end of Sec. 3.3.2, we are aware of at least one important special case in which identifiability holds globally in all of the parameter space.

Corollary III.2 states that unknown parameters corresponding to Problem P1 (defined in Sec. II) are globally identifiable, if the magnetic field is different from zero in at least one place, with no requirement about gradient. In both cases, magnetometer scale factors ${ }_{j}^{\mathrm{b}} \mathrm{a}$ are only identifiable up to an unknown global scale factor $\lambda$, while the magnetic field $\left({ }^{\mathrm{n}} \mathbf{B}\right)$ is identifiable up to the unknown global scale factor $1 / \lambda$.

In practical applications, measurements are not known for all 6D positions: only a finite set of sampled measurements are provided. In the following, we show that in practice, identifiability is preserved in this situation.

\section{Practical identifiability of parameters for simulated data}

In this section, we want to check if for a given realistic input, the above calibration problem can be 
solved. We proceed by collecting motion capture data in order to get a realistic sampled trajectory. We denote ${ }^{\mathrm{n}} \mathbf{M}[k]$ the sampled positions, and $R[k]$ the sampled attitudes, where $k$ is the sample number. For the purpose of the simulation, we choose at random a realistic set of parameters $\left(\left({ }_{j} b\right),\left({ }_{j}^{\mathrm{b}} \mathbf{p}\right),\left({ }_{j}^{\mathrm{b}} \mathbf{a}\right),\left(\alpha_{i}\right)\right)$, describing magnetometer calibration parameters and the magnetic field in the volume of the trajectory. Then we generate sampled values of measurements ${ }_{j} y[k]$ according to (2) at each trajectory sample,

$$
{ }_{j} y[k]={ }_{j}^{\mathrm{b}} \mathbf{a} \cdot R[k]^{\top} \cdot{ }^{\mathrm{n}} \mathbf{B}_{\left(\alpha_{i}\right)}\left({ }^{\mathrm{n}} \mathbf{M}[k]+R[k] \cdot{ }_{j}^{\mathrm{b}} \mathbf{p}\right)+{ }_{j} b .
$$

Problem P2 is rewritten as: given the set of sampled measurements ${ }_{j} y[k]$ and the sampled trajectory ${ }^{\mathrm{n}} \mathbf{M}[k]$, $R[k]$, find the exact set of parameters that has been used as input.

\subsection{Scale factor ambiguity}

Let us assume that there exists a tuple of parameters $\left(\left({ }_{j} b\right),\left({ }_{j}^{\mathrm{b}} \mathbf{p}\right),\left({ }_{j}^{\mathrm{b}} \mathbf{a}\right),\left(\alpha_{i}\right)\right)$ satisfying (9). Let $\lambda>0$. Then,

$$
{ }_{j} y[k]=\lambda_{j}^{\mathrm{b}} \mathbf{a} \cdot R[k]^{\top} \cdot \frac{1}{\lambda}{ }^{\mathrm{n}} \mathbf{B}_{\left(\alpha_{i}\right)}\left({ }^{\mathrm{n}} \mathbf{M}[k]+R[k] \cdot{ }_{j}^{\mathrm{b}} \mathbf{p}\right)+{ }_{j} b .
$$

Thus a tuple of parameters containing $\frac{1}{\lambda}{ }^{\mathrm{n}} \mathbf{B}_{\left(\alpha_{i}\right)}$ and $\lambda_{j}^{\mathrm{b}} \mathbf{a}$ is indistinguishable from the former tuple using only measurements ${ }_{j} y[k]$, as discussed in Sec. III. Therefore, in the rest of the document, we impose the global scale factor by setting the first coefficient of the scale factor of the first magnetometer to unity, ${ }_{1}^{\mathrm{b}} a_{1}=1$.

\subsection{Least-square parameter estimation model}

Let us denote magnetic measurement residuals ${ }_{j} r[k]$ such that for all $j$ and $k$,

$$
{ }_{j} r[k] \doteq{ }_{j}^{\mathrm{b}} \mathbf{a} \cdot R[k]^{\top} \cdot{ }^{\mathrm{n}} \mathbf{B}_{\left(\alpha_{i}\right)}\left({ }^{\mathrm{n}} \mathbf{M}[k]+R[k] \cdot{ }_{j}^{\mathrm{b}} \mathbf{p}\right)+{ }_{j} b-{ }_{j} y[k]
$$

By Eq. (9) for all $j, k,{ }_{j} r[k]=0$, and is equivalent to

$$
J_{\mathrm{mag}} \doteq \sum_{j, k}\left({ }_{j} r[k]\right)^{2}=0
$$

It follows that the problem could be seen as a generic non-linear least-square problem. Identifiability of parameters $\left(\left({ }_{j} b\right),\left({ }_{j}^{\mathrm{b}} \mathbf{p}\right),\left({ }_{j}^{\mathrm{b}} \mathbf{a}\right),\left(\alpha_{i}\right)\right)$ can be assessed on a specific trajectory by computing the rank of the Hessian matrix of the cost function $J_{\text {mag }}$ with respect to these unknown variables. Parameter estimation can be attempted by running a non-linear least-square numerical solver.

We propose using Ceres-Solver [25] and its implementation of the Levenberg-Marquardt algorithm in order to solve this calibration problem, taking advantage of its auto-differentiation framework. Doing so, we successfully show that the solver converges toward the correct parameter values, and numerically obtain that the Hessian of $J_{\mathrm{mag}}$ with respect to all estimated parameters is full rank in tested cases.

\subsection{Convergence of the Levenberg-Marquardt algorithm for simulated data}

Using an input trajectory, after choosing a set of calibration and magnetic field parameters and simulating corresponding measurements without noise, we choose a starting point at random, different from the set of calibration and magnetic field parameters used to simulate magnetic measurements, and run our non-linear least-square solver [25] implementing the Levenberg-Marquardt algorithm [26, 27]. This numerical algorithm is a common choice for solving non-linear least-square problems. In order to impose the global scale factor corresponding to the unit of the magnetic field, ${ }_{1}^{\mathrm{b}} a_{1}=1$ is excluded from the algorithm. Figure 1 shows the evolution of calibration parameter estimation errors at each iteration for all calibration parameters in both Problem P1 and Problem P2. In both cases, the solver converges successfully toward the correct solution, and stops iterating after the seventh iteration upon reaching its convergence criteria. Final errors are at most one millionth of initial errors.

\subsection{Conclusion of simulation experiments}

The above experiments confirm that in practice, identifiability holds for simulated sampled data, along a realistic input trajectory.

\section{Real-world experiments}

We use an Optitrack ${ }^{\mathrm{TM}}$ motion capture equipment, see Fig. 2, providing trajectory data at $\sim 240 \mathrm{~Hz}$, to track the movement of a sensor board carrying magnetometers and micro-electro-mechanical inertial sensors. The sensor board is the same that was used in $[18,19,21,22]$. All sensors are sampled at $f \approx 325 \mathrm{~Hz}$. No synchronization information between motion capture data and sensor board data is available in our setup. 


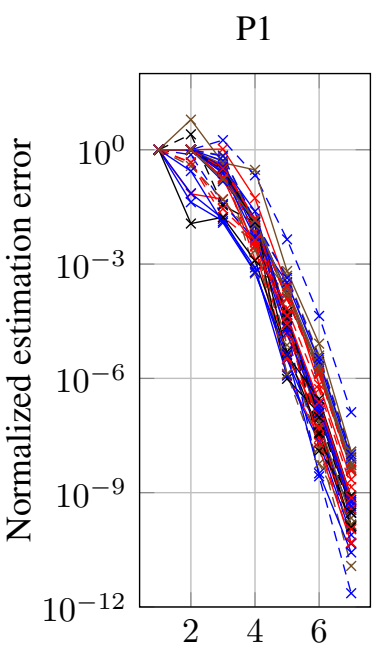

Iteration number

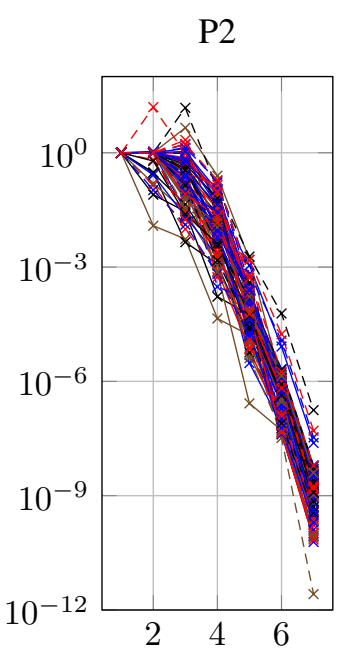

Iteration number
Fig. 1. Evolution of calibration parameters estimation error, with respect to iteration number for simulated data for Problem P1 (left) and Problem P2 (right). Errors converge toward 0. The solver reaches its convergence criteria and stops iterating at the seventh iteration in both cases.

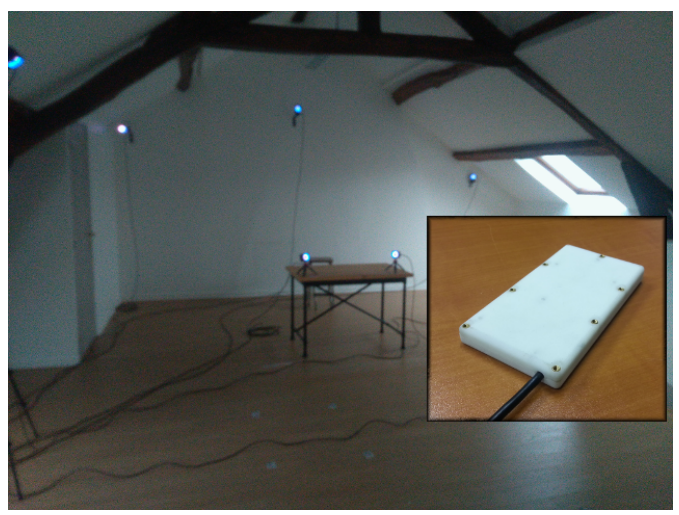

Fig. 2. Picture of the optitrack setup. The sensor board package is displayed in the insert.

Motion capture data is noisy and not synchronized with sensor board data. Also, body frames are not necessarily defined in the same way for each type of information (gyrometer, accelerometer, magnetometer and motion capture). To account for different measurement uncertainties we use three sets of equations: integrated discrete-time strapdown IMU navigation equations, as can be found in [28, Ch. 7], simplified motion capture measurement equations, and magnetic measurement equations corresponding to (9). The augmented problem is then reformulated as a non-linear least-squares identification problem in the same manner as in (11) and (12). Moreover, magnetic

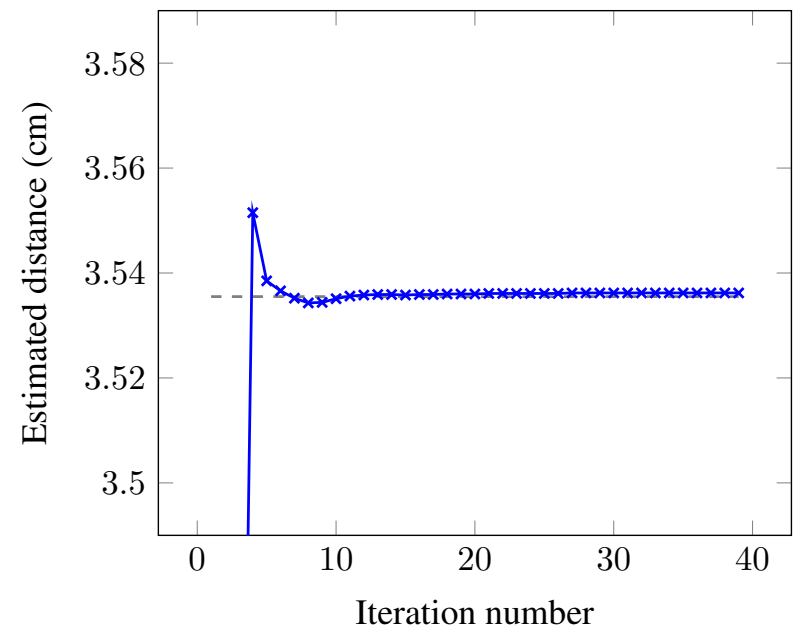

Fig. 3. Evolution of the estimated distance between two magnetic sensors of the array as a function of algorithm iteration number (blue solid line). All positions are initialized as $0 \mathrm{~cm}$. The distance by construction can be expected to be $35.4 \mathrm{~mm} \pm 0.2 \mathrm{~mm}$ (gray dashed line). The final estimated distance is $35.36 \mathrm{~mm}$.

measurements are filtered in order to remove power-line interference.

The sensor board is moved around in a space covered by the motion capture setup. A sufficiently diverse arbitrary trajectory is chosen, with enough rotations and translations, in an indoor magnetic field satisfying identifiability hypotheses for Problem P2 (defined in Sec. II). Sensor and motion capture data is collected, and injected into the optimization problem that is solved using Ceres-Solver [25] as described in Sec IV.

The solver converges toward a set of scale factors and biases that are in agreement with those obtained using the technique in [1]. The estimated positions are coherent with sensor-sensor distances expected by construction (see Fig. 3). With these results, we have verified that identifiability also holds in real-world experiments. ${ }^{\ddagger}$

\section{Conclusion}

This paper has demonstrated that magnetic data together with position and attitude information suffices to identify calibration parameters of linearresponse single-axis point magnetometers, including their effective positions within the device. Experimental data has confirmed the identifiability we have proven with a mathematical theory in real situations. This

\footnotetext{
${ }^{\ddagger}$ A patent is pending on the whole calibration process.
} 
approach makes it possible to calibrate arrays of magnetometers in magnetic fields within unknown environments, provided that the magnetic field is stationary and not specifically homogeneous, and that positioning information is available. For instance, the underlying concept could be used to extend tools such as [29] to calibrate visual-inertial setups coupled with magnetic sensor arrays such as in [20,21, 22]. Finally, experimental results suggest that some positioning information might be redundant. In a subsequent study, we intend to understand what minimal information is needed in order to identify calibration parameters.

\section{A. Proof of Lemma III.3}

We adopt the assumptions and notations of Sec. 3.3.1. Let $j \in\{1,2, \ldots, m\}$, and ${ }^{\mathrm{n}} \mathbf{M} \in \mathbb{R}^{3}$. Inserting Eq. (3) into the integral and subtracting the bias ${ }_{j} b$ from it, we find

$$
\begin{aligned}
& \oint_{R \in \mathrm{SO}(3)} y\left({ }_{j}^{\mathrm{n}} \mathbf{M}, R\right) \mathrm{d} R-{ }_{j} b \\
= & \oint_{R \in \mathrm{SO}(3)}{ }_{j}^{\mathrm{b}} \mathbf{a} \cdot R(t)^{\top} \cdot{ }^{\mathrm{n}} \mathbf{B}\left({ }^{\mathrm{n}} \mathbf{M}+R \cdot{ }_{j}^{\mathrm{b}} \mathbf{p}\right) \mathrm{d} R,
\end{aligned}
$$

as $\mathrm{d} R$ is normalized such that the volume of $\mathrm{SO}(3)$ is unity. In order to prove the lemma, it suffices to show that the expression (13) vanishes.

To do so, we recall that any element $R \in \mathrm{SO}(3)$ can be decomposed (see also Fig. 4) into two rotations, $R=R_{2} \cdot R_{1}$, such that the first rotation $R_{1}$ in $\mathcal{S}^{1} \cong$ $\mathrm{SO}(2))$ is around ${ }_{j}^{\mathrm{b}} \mathbf{p}$ and the second rotation $R_{2}$ in $\mathcal{S}^{2}(\cong \mathrm{SO}(3) / \mathrm{SO}(2))$ around an axis orthogonal to ${ }_{j}^{\mathrm{b}} \mathbf{p}$. The first rotation leaves the sensor position constant, which means that ${ }^{\mathrm{n}} \mathbf{M}+R \cdot{ }_{j}^{\mathrm{b}} \mathbf{p}={ }^{\mathrm{n}} \mathbf{M}+R_{2} \cdot{ }_{j}^{\mathrm{b}} \mathbf{p}$ does not depend on $R_{1}$. Then,

$$
\begin{aligned}
& \oint_{R \in \mathrm{SO}(3)}{ }_{j}^{\mathrm{b}} \mathbf{a} \cdot R(t)^{\top} \cdot{ }^{\mathrm{n}} \mathbf{B}\left({ }^{\mathrm{n}} \mathbf{M}+R \cdot{ }_{\cdot j}^{\mathrm{b}} \mathbf{p}\right) \mathrm{d} R \\
= & \oint_{R_{2} \in \mathcal{S}^{2}}\left[\oint_{R_{1} \in \mathcal{S}^{1}}{ }_{j}^{\mathrm{b}} \mathbf{a} \cdot R_{1}^{\top} \mathrm{d} R_{1}\right] \cdot R_{2}^{\top \mathrm{n}} \cdot{ }^{\mathrm{n}} \mathbf{B}\left({ }^{\mathrm{n}} \mathbf{M}+R_{2} \cdot{ }_{j}^{\mathrm{b}} \mathbf{p}\right) \mathrm{d} R_{2}
\end{aligned}
$$

By construction (cf. also Fig. 4), the integral over $R_{1}$ is proportional to ${ }_{j}^{\mathrm{b}} \mathbf{p}^{\top}$. Thus, the expression (13) is proportional to

$$
\oint_{R_{2} \in \mathcal{S}^{2}}\left(R_{2} \cdot{ }_{j}^{\mathrm{b}} \mathbf{p}\right)^{\top} \cdot{ }^{\mathrm{n}} \mathbf{B}\left({ }^{\mathrm{n}} \mathbf{M}+R_{2} \cdot{ }_{j}^{\mathrm{b}} \mathbf{p}\right) \mathrm{d} R_{2} .
$$

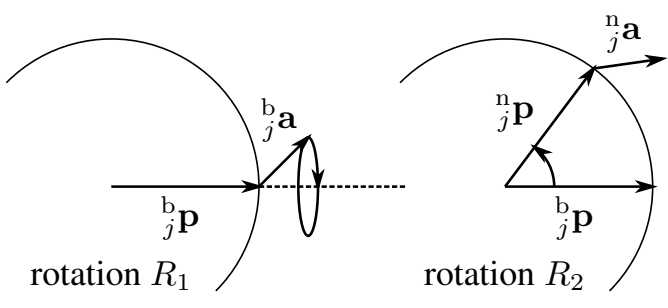

Fig. 4. A rotation $R \in \mathrm{SO}(3)$ can be decomposed as $R=R_{2} . R_{1}$ where $R_{1}$ rotates around ${ }_{j}^{\mathrm{b}} \mathbf{p}$, and $R_{2}$ rotates around an axis orthogonal to ${ }_{j}^{\mathrm{b}} \mathbf{p}$.

This expression is just the magnetic flux through the surface of a sphere of radius $\left\|{ }_{j}^{\mathrm{b}} \mathbf{p}\right\|$. According to Gauss' theorem for magnetism, it must vanish. As a result, the expression (13) vanishes indeed, which concludes the proof.

\section{B. Proof of Lemma III.4}

We adopt assumptions and notations of Sec. 3.3.2. Without loss of generality, we may put

$$
{ }_{j 0}^{\mathrm{b}} \mathbf{p}=0 \text {, }
$$

which can always be achieved by redefining the center of the moving frame $\Re_{b}$. In this case, the injective mapping $\zeta$ to be constructed has to satisfy $0=\zeta^{-1}(0)$.

In order to facilitate the construction, let

$$
\begin{aligned}
& S_{\phi} \doteq\left(\begin{array}{ccc}
\cos \phi & -\sin \phi & 0 \\
\sin \phi & \cos \phi & 0 \\
0 & 0 & 1
\end{array}\right), \\
& T_{\phi} \doteq\left(\begin{array}{ccc}
\cos \phi & 0 & \sin \phi \\
0 & 1 & 0 \\
-\sin \phi & 0 & \cos \phi
\end{array}\right), \\
& U_{\phi} \doteq\left(\begin{array}{ccc}
1 & 0 & 0 \\
0 & \cos \phi & -\sin \phi \\
0 & \sin \phi & \cos \phi
\end{array}\right)
\end{aligned}
$$

with $\phi \in[0,2 \pi]$. For fixed ${ }^{\mathrm{n}} \mathbf{M} \in \mathbb{R}^{3}, R \in \mathrm{SO}(3)$ and given measurement function ${ }_{j} y$, we define the mapping

$$
\begin{aligned}
& \zeta: \mathbb{R}^{3} \rightarrow \mathbb{R}^{6} \\
& \mathbf{q} \mapsto \frac{1}{2} \sum_{k=0}^{3}(-1)^{k}\left(\begin{array}{l}
\left({ }_{j} y \circ f\right)\left(\mathbf{q},{ }^{\mathrm{n}} \mathbf{M}, R \cdot S_{\frac{k \pi}{2}}\right) \\
\left({ }_{j} y \circ f\right)\left(\mathbf{q},{ }^{\mathrm{n}} \mathbf{M}, R \cdot S_{\frac{k \pi}{2}+\frac{\pi}{4}}\right) \\
\left({ }_{j} y \circ f\right)\left(\mathbf{q},{ }^{\mathrm{n}} \mathbf{M}, R \cdot T_{\frac{k \pi}{2}}\right) \\
\left({ }_{j} y \circ f\right)\left(\mathbf{q},{ }^{\mathrm{n}} \mathbf{M}, R \cdot T_{\frac{k \pi}{2}+\frac{\pi}{4}}\right) \\
\left({ }_{j} y \circ f\right)\left(\mathbf{q},{ }^{\mathrm{n}} \mathbf{M}, R \cdot U_{\frac{k \pi}{2}}\right) \\
\left({ }_{j} y \circ f\right)\left(\mathbf{q},{ }^{\mathrm{n}} \mathbf{M}, R \cdot U_{\frac{k \pi}{2}+\frac{\pi}{4}}\right)
\end{array}\right)
\end{aligned}
$$



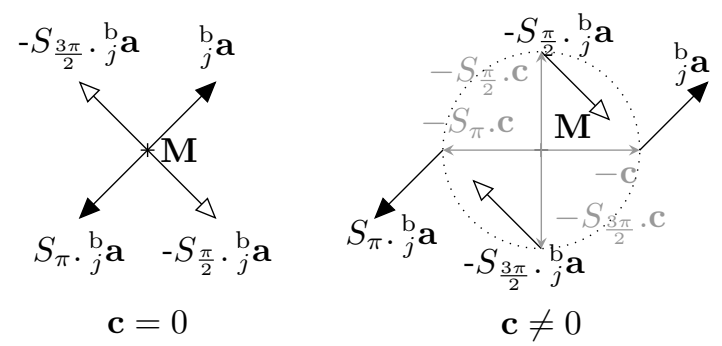

Fig. 5. (left) The first component of $\zeta(0)$ can be expressed as an inner product between the magnetic field at $\mathbf{M}$ and ${ }_{j}^{\mathrm{b}} \mathbf{a} \cdot\left(\sum_{k=0}^{3}(-1)^{k} S_{\frac{k \pi}{2}}\right)^{\top}=0$, which is always 0 . (right) At $\mathbf{q} \neq 0, \zeta(\mathbf{q})$ becomes dependent on spatial variations of the magnetic field around $\mathbf{M}$. The construction is analogous for other components.

with

$$
\begin{aligned}
f: \mathbb{R}^{3} \times \mathbb{R}^{3} \times \mathrm{SO}(3) & \rightarrow \mathbb{R}^{3} \times \mathrm{SO}(3) \\
\left(\mathbf{q},{ }^{\mathrm{n}} \mathbf{M}, R\right) & \mapsto\left({ }^{\mathrm{n}} \mathbf{M}-R . \mathbf{q}, R\right)
\end{aligned} .
$$

By definition of $f$ and ${ }_{j} y$, i.e. (3) and (20), and using assumption (15),

$$
\left({ }_{j} y \circ f\right)\left(\mathbf{q},{ }^{\mathrm{n}} \mathbf{M}, R\right)=\left(R \cdot{ }_{j}^{\mathrm{b}} \mathbf{a}\right) \cdot{ }^{\mathrm{n}} \mathbf{B}\left({ }^{\mathrm{n}} \mathbf{M}-R . \mathbf{q}\right) .
$$

To prove the lemma, we first show that $\zeta(0)=0$ independently of scale factors and the magnetic field. For $\mathbf{q}=0$, we find that the first component of $\zeta(\mathbf{q})$ is proportional to $\sum_{k=0}^{3}(-1)^{k} S_{\frac{k \pi}{2}}^{\top}=0$, cf. also Fig. 5. A completely analogous calculation shows that in fact all components of $\zeta(q)$ vanish for $\mathbf{q}=0$, independently of other parameters.

In order to show injectivity, consider the linear approximation in $\mathbf{q}$,

$$
\begin{aligned}
& \left({ }_{j} y \circ f\right)\left(\mathbf{q},{ }^{\mathrm{n}} \mathbf{M}, R\right) \\
& \simeq{ }_{j}^{\mathrm{a}} \mathbf{a} \cdot R^{\top}{ }^{\mathrm{n}} \mathbf{B}\left({ }^{\mathrm{n}} \mathbf{M}\right)-\left.{ }_{j}^{\mathrm{b}} \mathbf{a} \cdot R^{\top} \cdot \nabla{ }^{\mathrm{n}} \mathbf{B}\right|_{{ }_{\mathrm{n}} \mathbf{M}} \cdot R \cdot \mathbf{q} .
\end{aligned}
$$

By assumption, there exists a position ${ }^{n} \mathbf{M}_{0}$ such that the magnetic gradient's eigenvalues are different from each other. Also, since Ampere's law in the absence of source currents implies that $\left.\nabla^{\mathrm{n}} \mathbf{B}\right|_{{ }_{n} M_{0}}$ is symmetric, its matrix can be diagonalized by an orthogonal matrix. Thus, there exists $R_{0} \in \mathrm{SO}(3)$ such that

$$
\left(\begin{array}{ccc}
d_{1} & 0 & 0 \\
0 & d_{2} & 0 \\
0 & 0 & d_{3}
\end{array}\right)=\left.R_{0}^{\top} \cdot \nabla^{\mathrm{n}} \mathbf{B}\right|_{\mathrm{n}_{\mathbf{M}}} \cdot R_{0},
$$

with mutually different $d_{1}, d_{2}, d_{3} \in \mathbb{R}$.

We now specialize the construction of the mapping $\zeta$, Eq. (19), by inserting the $6 \mathrm{D}$ position $\left({ }^{\mathrm{n}} \mathbf{M}_{0}, R_{0}\right)$ for ${ }^{\mathrm{n}} \mathbf{M}$ and $R$. Using the notation ${ }_{j}^{\mathrm{b}} \mathbf{a}=$ $\left(\begin{array}{lll}{ }_{j}^{\mathrm{b}} a_{1} & { }_{j}^{\mathrm{b}} a_{2} & { }_{j}^{\mathrm{b}} a_{3}\end{array}\right)$, we find that the Jacobian matrix of the mapping $\zeta$, Eq. (19), at $\mathbf{q}=0$ takes the form

$$
\begin{aligned}
& \left.\nabla \zeta\right|_{0} \\
& =\left(\begin{array}{ccc}
-\left(d_{1}-d_{2}\right)_{j}^{\mathrm{b}} a_{1} & \left(d_{1}-d_{2}\right)_{j}^{\mathrm{b}} a_{2} & 0 \\
\left(d_{1}-d_{2}\right)_{j}^{\mathrm{b}} a_{2} & \left(d_{1}-d_{2}\right)_{j}^{\mathrm{b}} a_{1} & 0 \\
\left(d_{3}-d_{1}\right)_{j}^{\mathrm{b}} a_{1} & 0 & -\left(d_{3}-d_{1}\right)_{j}^{\mathrm{b}} a_{3} \\
\left(d_{3}-d_{1}\right)_{j}^{\mathrm{b}} a_{3} & 0 & \left(d_{3}-d_{1}\right)_{j}^{\mathrm{b}} a_{1} \\
0 & -\left(d_{2}-d_{3}\right)_{j}^{\mathrm{b}} a_{2} & \left(d_{2}-d_{3}\right)_{j}^{\mathrm{b}} a_{3} \\
0 & \left(d_{2}-d_{3}\right)_{j}^{\mathrm{b}} a_{3} & \left(d_{2}-d_{3}\right)_{j}^{\mathrm{b}} a_{2}
\end{array}\right) .
\end{aligned}
$$

Since by assumption, the $d_{1}, d_{2}, d_{3}$ are mutually different and ${ }_{j}^{\mathrm{b}} \mathbf{a} \neq 0$, the $6 \times 3$ Jacobian matrix $\left.\nabla \zeta\right|_{0}$ contains necessarily a non-zero minor determinant of order 3 , and thus is injective. As a result, the mapping $\zeta$ itself is an immersion in $\mathbf{q}=0$, and thus injective, at least locally, around $\mathbf{q}=0$.

\section{REFERENCES}

1. E. Dorveaux, D. Vissière, A.-P. Martin, and N. Petit, "Iterative calibration method for inertial and magnetic sensors," in Proceedings of the 48h IEEE Conference on Decision and Control (CDC) held jointly with 2009 28th Chinese Control Conference, (Shanghai, China), pp. 8296-8303, dec 2009.

2. V. Renaudin, M. Afzal, and G. Lachapelle, "Complete triaxis magnetometer calibration in the magnetic domain," Journal of Sensors, vol. 2010, pp. 1-10, 2010.

3. X. Li, Y. Wang, and Z. Li, "Calibration of tri-axial magnetometer in magnetic compass using vector observations," in 2015 IEEE 28th Canadian Conference on Electrical and Computer Engineering (CCECE), IEEE, may 2015.

4. M. Kok, J. D. Hol, T. B. Schön, F. Gustafsson, and $\mathrm{H}$. Luinge, "Calibration of a magnetometer in combination with inertial sensors," in 2012 15th International Conference on Information Fusion, (Singapore), pp. 787-793, July 2012.

5. D. Yang, Z. You, B. Li, W. Duan, and B. Yuan, "Complete tri-axis magnetometer calibration with a gyro auxiliary," Sensors, vol. 17, no. 6, p. 1223, 2017.

6. K. Mohamadabadi and M. Hillion, "An automated indoor scalar calibration method for three-axis vector magnetometers," IEEE Sensors Journal, vol. 14, pp. 3076-3083, sep 2014.

7. M. Díaz-Michelena, R. Sanz, M. F. Cerdán, and A. B. Fernández, "Calibration of qmmoura three-axis magnetometer and gradiometer," 
Geoscientific Instrumentation, Methods and Data Systems, vol. 4, no. 1, pp. 1-18, 2015.

8. H. Pang, S. Luo, Q. Zhang, J. Li, D. Chen, M. Pan, and F. Luo, "Calibration of a fluxgate magnetometer array and its application in magnetic object localization," Measurement Science and Technology, vol. 24, p. 075102, may 2013.

9. S. Turner, M. J. Hall, S. Harmon, and N. Hillier, "Calibration of a novel three-axis fluxgate gradiometer for space applications," IEEE Transactions on Magnetics, vol. 51, pp. 1-4, jan 2015.

10. Y. Adachi, M. Higuchi, D. Oyama, Y. Haruta, S. Kawabata, and G. Uehara, "Calibration for a multichannel magnetic sensor array of a magnetospinography system," IEEE Transactions on Magnetics, vol. 50, pp. 1-4, nov 2014.

11. T. Yoshida, M. Higuchi, T. Komuro, and H. Kado, "Calibration system for a multichannel squid magnetometer," in Proceedings of 16th Annual International Conference of the IEEE Engineering in Medicine and Biology Society, (Baltimore, MD, USA), nov 1994.

12. D. Vissière, A. Martin, and N. Petit, "Using distributed magnetometers to increase imu-based velocity estimation into perturbed area," in Conference on Decision and Control (CDC), (New Orleans, LA), pp. 4924-4931, 2007.

13. D. Vissière, A. Martin, and N. Petit, "Système fournissant la vitesse et la position d'un corps en utilisant les variations du champ magnétique évaluées grâce aux mesures de un ou des magnétomètres et de une ou des centrales inertielles," Patent FR2914739 (A1), 2008.

14. E. Dorveaux, Magneto-inertial navigation: principles and application to an indoor pedometer. $\mathrm{PhD}$ thesis, Ecole Nationale Supérieure des Mines de Paris, France, 2011.

15. E. Dorveaux, T. Boudot, M. Hillion, and N. Petit, "Combining inertial measurements and distributed magnetometry for motion estimation," in American Control Conference (ACC), (San Francisco, CA), pp. 4249-4256, 2011.

16. E. Dorveaux and N. Petit, "Presentation of a magneto-inertial positioning system: navigating through magnetic disturbances," in International Conference on Indoor Positioning and Indoor Navigation (IPIN), (Guimarães, Portugal), 2011.

17. N. Praly, P.-J. Bristeau, J. Laurent-Varin, and N. Petit, "Using distributed magnetometry in navigation of heavy launchers and space vehicles," in Progress in Flight Dynamics, Guidance, Navigation, Control, Fault Detection, and Avionics, vol. 6, pp. 45-54, EDP Sciences, 2013.
18. C.-I. Chesneau, M. Hillion, and C. Prieur, "Motion estimation of a rigid body with an EKF using magneto-inertial measurements," in 2016 International Conference on Indoor Positioning and Indoor Navigation (IPIN), (Alcalá de Henares, Spain), oct 2016.

19. C.-I. Chesneau, M. Hillion, J.-F. Hullo, G. Thibault, and C. Prieur, "Improving magnetoinertial attitude and position estimation by means of a magnetic heading observer," in 2017 International Conference on Indoor Positioning and Indoor Navigation (IPIN), (Sapporo, Japan), sep 2017.

20. D. Caruso, A. Eudes, M. Sanfourche, G. Le Besnerais, and D. Vissière, "Infrastructureless indoor navigation with an hybrid magneto-inertial and depth sensor system," in 2016 International Conference on Indoor Positioning and Indoor Navigation (IPIN), (Alcalá de Henares, Spain), oct 2016.

21. D. Caruso, A. Eudes, M. Sanfourche, D. Vissière, and G. Le Besnerais, "A robust indoor/outdoor navigation filter fusing data from vision and magneto-inertial measurement unit," Sensors, vol. 17, p. 2795, dec 2017.

22. D. Caruso, A. Eudes, M. Sanfourche, D. Vissière, and G. Le Besnerais, "Robust indoor/outdoor navigation through magneto-visual-inertial optimization-based estimation," in 2017 IEEE/RSJ International Conference on Intelligent Robots and Systems (IROS), (Vancouver, Canada), sep 2017.

23. J. Jackson, Classical Electrodynamics, Third Edition. John Wiley \& Sons, Inc., 1998.

24. S. Willard, General Topology. Dover Publications, Inc., 2004.

25. S. Agarwal, K. Mierle, and Others, "Ceres solver." http://ceres-solver.org.

26. K. Levenberg, "A method for the solution of certain non-linear problems in least squares," Quarterly of Applied Mathematics, vol. 2, pp. 164-168, jul 1944.

27. D. W. Marquardt, "An algorithm for least-squares estimation of nonlinear parameters," Journal of the Society for Industrial and Applied Mathematics, vol. 11, pp. 431-441, jun 1963.

28. P. G. Savage, Strapdown Analytics by Paul G. Savage. Strapdown Associates, 2000.

29. P. Furgale, J. Rehder, and R. Siegwart, "Unified temporal and spatial calibration for multi-sensor systems," in Intelligent Robots and Systems (IROS), 2013 IEEE/RSJ International Conference on, pp. 1280-1286, 2013. 